\title{
How Effective are Injections of Platelet-Rich Plasma (PRP) for the Treatment of Sports Injuries: a Critical Review of the Literature
}

\section{JL Ziltener*, L Allet, P Sclison and M Grosclaude}

Geneva University Hospital, Geneva, Switzerland

\begin{abstract}
Acute soft-tissue lesions and chronic overuse injuries in the sports medicine field are very frequent and in most cases, difficult to treat. Tissue repair in musculoskeletal lesions is often too slow and sometimes incomplete for a given athlete. The fastest, most complete recovery is of primary importance, and keeping the delay until return-tofield as brief as possible, a priority. Many bioactive proteins and growth factors amongst others influence healing processes. Administration of Platelet Rich Plasma enables the production, from the patient's own blood, of natural, high concentrations of autologous growth factors. Basic research and animal studies are promising, but evidencebased studies examining the treatment of human musculoskeletal lesions by Platelet Rich Plasma are still lacking. Nevertheless, such treatment is increasingly used in sports medicine clinical practice and much is expected of growth factor injections. The aim of this non exhaustive review is to analyze the existing literature published in the fields of sports medicine. The results of this analysis do not permit us to recommend the systematic and generalized use of Platelet Rich Plasma injections in the management of sport injuries. Nevertheless, a few promising results have to be mentioned, but high-quality studies are needed to provide scientific evidence about these injections' effectiveness.
\end{abstract}

\section{Introduction}

Acute and chronic musculoskeletal injuries are common during physical activities, are frequently problematic for an athlete, and always represent a therapeutic challenge for the clinician. Tissue healing after such injuries may be slow and sometimes incomplete. Nevertheless, optimal treatment for these injuries is mandatory and should be as safe, quick and cost-effective as possible, in order to restore athletes' preinjury level in sports participation.

Physiologically, the healing of an injury progresses in overlapping phases and is characterized by a cascade of events triggered by the injured tissues. The three classic phases are: inflammation, proliferation and remodeling.

There are several treatment strategies with which to address musculoskeletal injuries. One novel approach is intra-lesion, autologous blood injection. Several products are available: Autologous Conditioned Serum (ACS), Platelet-Rich Plasma (PRP), Plasma Rich in Growth Factors (PRGF) and other derivatives. The large variety of products speaks for their different possible compositions with diverse biological effects. Other variables such as modalities of PRP activation, presence or not of white blood cells, local anesthesia or not, use of non-steroidal anti-inflammatory drugs, post-injection rehabilitation protocols, and many others, are confounding factors in studies, explaining the diversity of the experimental, but most of all clinical results $[1,2]$.

PRP is a simple and minimally invasive means of obtaining the concentration of autologous growth factors. It is defined as a fraction of blood plasma, containing higher concentration of platelets than normal plasma, and consequently, a source of growth factors. Blood platelets consist of fifty to eighty alpha granula per unit. Each granula has more than thirty bioactive proteins, including platelet-derived growth factor (PDGF), transforming growth factor (TGF-Beta), vascular endothelial growth factor (VEGF), fibroblastic growth factor (FGF), epidermal growth factor (EGF), and insulin-like growth factor 1 (IGF-1). The different effects these factors have on human tissues have been extensively discussed in numerous papers, synthesized in two recent reviews $[3,4]$

PRP is directly injected in the lesion, mostly under echo graphic control. PRP thus supports tissue regeneration by acting as a biological catalyst. In addition, it seems to show antibacterial characteristics [5].
PRP is neither considered as medication, nor as a therapeutic substance. Hence, the rules of its application remain vague. Furthermore, its use is no longer banned by the world anti-doping agency, regardless of the chosen application procedure. Administration of PRP has been rapidly increasing since 2005 (the year the first clinical study involving injured athletes was published), probably due to its low price and efforts by the chemical industry. The world of sports is interested in any therapeutic approach that may promote the speedy recovery of injured athletes. In order to specify the clinical use of PRP, an international consensus covering all domains of sports medicine has been published by the International Olympic Committee [3].

Basic research and animal studies on the subject are relatively promising. Nevertheless, there is still ample discussion about the effectiveness of platelet-rich plasma injections for the treatment of human acute, or chronic, musculoskeletal injuries. This paper provides a critical, but not exhaustive, overview of the effect of platelet-rich plasma injections on tendon, muscle or ligament lesions, described in experimental and animal studies. We focused in particular in human in-vivo investigations.

\section{Methodology}

To assess current evidence for autologous plasma injections in the management of not surgically treated musculoskeletal injuries, a literature search using EMBASE and MEDLINE databases was conducted. We didn't extend our search to other databases, for example those including non-English articles. The key words were (autologous conditioned serum OR platelet-rich plasma) AND

*Corresponding author: Jean Luc Ziltener, Geneva University Hospital, Geneva, Switzerland, E-mail: Jean-Luc.Ziltener@hcuge.ch

Received December 22, 2011; Accepted February 06, 2012; Published February 10,2012

Citation: Ziltener JL, Allet L, Sclison P, Grosclaude M (2012) How Effective are Injections of Platelet-Rich Plasma (PRP) for the Treatment of Sports Injuries: a Critical Review of the Literature. J Sport Medic Doping Studie S2:003. doi:10.4172/2161-0673.S2-003

Copyright: (C) 2012 Ziltener JL, et al. This is an open-access article distributed under the terms of the Creative Commons Attribution License, which permits unrestricted use, distribution, and reproduction in any medium, provided the original author and source are credited. 
(animals OR Fundamentals OR Human studies) AND (soft tissuesmuscle-ligament-tendon injuries). All titles were checked for the presence of a reference to autologous plasma injections. All articles with the said reference were then classified, based on their abstract, into fundamental or non fundamental studies, the latter group being subdivided in animal or human studies. At this time of selection, we identified 74 studies, 52 concerning animal research and 22 for human trials. Two persons read the articles and selected the studies considered methodologically acceptable following the Judet criteria.

As a particular focus was paid on human investigations we were interested in the quality of these trials. Among all identified human trials, only seven articles reached an evidence level type I or II (three studies referred to anterior cruciate ligament and four other were concerning tendon injuries). The quality of these studies was evaluated with the Pedro Scale. The quality ranged between $6 / 10$ and $9 / 10$ points. Given the paucity of high-level evidence based articles (level I and II) concerning soft tissue injuries, we finally decided to check the references of recent literature reviews on the subject. However, no additional article could be identified.

This article provides an overview and update destined to primary care physicians and sports medicine specialists. It should help them to better evaluate the risks and benefits inherent to PRP injections.

\section{Platelet-Rich Plasma and Fundamental Sciences - Animal Studies}

\section{PRP and tendon}

PRP is a relatively well established treatment option for the management of chronic tendon injuries, and its beneficial effects have been demonstrated in various animal studies listed in several recent reviews $[2,3,6]$.

A Japanese study tested the effect of PRP in rats with a patella tendon lesion. In this paper, fibroblast cells and bone marrow stem cells in, and around the lesion were found to increase. Additionally, cell proliferation was twice as high, seven days post injection, as three days post injection. The group treated with PRP also showed a significant increase in collagen type I and III compared to the control group [7].

Another study conducted in-vitro cultures of equine tenocytes. Tendons that had been placed in a culture of PRP showed an increase in the genetic expression of mRNA for collagen type I and III, without an increase of metalloproteinase concentration or of catabolic proteins (MMP-13, MMP-3). An optimal concentration of PRP seems to be the determinant factor for increasing the expression of pro-collagen factors, but not the genetic expression of pro-inflammatory factors [8].

PRP increased cellular proliferation and the production of collagen in human tenocytes in vitro. The tenocytes in a PRP culture also increased the receptor expression for Vascular Endothelial Growth Factor A (VEGF-A). VEGF concentration was at its peak during the phases of proliferation and remodeling, and led to angiogenesis an important process of tissue healing. The Transforming Growth Factor-beta (TGF-B), highly concentrated in presence of PRP, played a predominant role in the tendon healing process. In the presence of a lesion, the number of TGF-B receptors on the tenocyte surface of rats (in vitro) had increased, which demonstrates the production of collagen Type I and III [9].

\section{PRP and ligaments}

Application of PRP is relatively well described for the anterior cruciate knee ligament in experimental and animal studies.
For example, in-vitro treatment of cells of the anterior cruciate ligament with PRP showed an increase of cells and collagen production [10].

Recently, in another in-vitro study conducted in mice, the association of basic Fibroblast Growth Factor (bFGF) and Growth Differentiation Factor 5 (GDF-5) increased cell proliferation, fibroblast migration, and the genetic expression of collagen Type I. The same study identified a better alignment of the medial collateral ligaments (MCL) collagen fibers compared to a control group [11].

It also has been shown that PDGF may play a significant role in the early stages of healing. Injected in the ruptured medial collateral ligament (MCL) of rabbits, PDGF improved biomechanical parameters such as ultimate load to failure, energy absorbed to failure and ultimate elongation of the MCL at 6 weeks [12].

A study conducted in rats found a $73 \%$ higher MCL ligament strength, compared to a control group who had no injection, at 12 days [13].

However, a study investigating the effect of PDGF on injured medial collateral ligament of rats, showed a significant decrease in its effectiveness when administered more than 24 hours after injury. This would suggest administering PRP preparation in the early phase for optimal healing [14].

Thus, basic science shows that the application of PRP stimulates the early healing of acute ligament injuries while improving the structure of injured ligaments.

\section{PRP and muscle}

Only a few experimental, in-vivo studies demonstrated the importance of growth factors for the healing process of muscle lesions, in terms of muscle regeneration and myogenesis. IGF-1, PDGF, NGF, FGF-2, HGF, and TGF-Beta seem to play an important role in muscle tissue healing. The last three, FGF-2, HGF, and TGFbeta, are particularly useful for the activation of satellite cells and are potent stimulators of the proliferation and fusion of myoblasts [15] For example, the injection of anti FGF-2 decreased the number and diameter of myofibers, and delayed the fusion activation of satellite cells [16].

Extrinsic muscle lesions in animals (shock-induced gastrocnemius muscle contusion in mice), if placed in an environment rich in growth factors, in particular FGF-2 and TGF-Beta, were shown to react positively. The application of autologous conditioned serum (ACS) activated satellite cells and triggered the proliferation and differentiation of myoblasts, with fusion in myotubes. WrightCarpenter et al. observed an increase in mature myofibers by day seven in a group that was treated with growth factors, compared to the control group. However, no significant difference could be identified by day fourteen, which only indicates an initial healing effect on muscle tissue, but not on muscle function, in the treated group [17].

More recently, PRP applied to a model of multiple, eccentrically loaded small muscle strains in rats, and caused a significant decrease in the total recovery time in the treated group, compared to non-treated rats. Functional improvement was significant in the PRP group from day seven and later at day 14 . In the same study with a model of a large strain injury, moderate functional improvement appeared at day three after injection, but not later on. Hence, in animal models of small eccentric muscle strains, PRP could improve muscle regeneration, but in presence of larger intrinsic muscle strains, results seem more doubtful [18]. 


\section{PRP in Human Chronic Tendinopathies}

Chronic tendinopathy is one of the most frequent lesions in musculoskeletal, sports injuries. Tendon problems are reputed to represent 30 to $50 \%$ of all sports injuries in both professional and hobby athletes. The tendons affected by lesions are primarily sportspecific.

Chronic tendinopathy is characterized by pain, edema and tendon weakness. Its origin is multi factorial. The repetition of micro traumas due to exercise, can lead to degenerative intra tendon lesions, micro ruptures, and sometimes to poor healing [19]. Histo-pathologically, these lesions present non-inflammatory cell proliferation (in particular fibroblasts), vascular hyperplasia (neo-vascularization), and disorganized collagen fibers, without any sign of inflammation [20,21].

In the tendon area, only a very few randomized controlled trials have been conducted in humans. Most of the studies are either cohort studies or case series. In this critical, non-exhaustive review, we focus on the three tendons most frequently affected in sports lesions: the Achilles tendon, patellar tendon, and tendons around the elbow ("epicondylitis"). These also happen to be the structures for which the PRP effect has been most investigated. It might be that we missed some interesting studies with our limited literature search strategy. However, the main reviews on the topic $[5,22,23]$ mention similar conclusions as we did.

\section{Achilles tendinopathy}

Only one double blind randomized controlled trial exists on this topic. PRP injections were compared with a saline isotonic solution in 54 patients that presented symptoms for at least two months. Concurrently, the subjects were admitted to a three month-long, eccentric exercise program. As a result of the treatment, pain decreased, and functional scores, such as VISA-A, improved significantly in both groups. Even if results in the PRP group were somewhat higher, no statistical significance was found after six months. There was also no difference in patient satisfaction or in the time taken to return to sport. Importantly, no relevant side effects were identified. The limitations of this study were the unique injection of the PRP, limited follow up time, the relatively old age of the participants, and the lack of a control group without an eccentric program [24].

The same investigators subsequently extended the follow up to one year, without finding any supplementary PRP benefit. Localized bleeding caused by the injecting syringe might have triggered the tissue healing process. A positive response could also be due to a placebo effect, as invasive procedures lead to higher expectancy of good results [25]. Finally, in both groups, ultrasound showed that tendon structure and neo-vascularization improved significantly, without any group differences after six and twelve months. One of the main reasons for the absence of group differences is certainly the fact that standardized eccentric exercise training has already been shown to improve actual tendon structure [26].

Another article, a prospective case series with fourteen patients, showed a significant improvement of functional scores for up to eighteen months after PRP injections. Echography further confirmed an improvement of structural anomalies. This study supports previous results, indicating a potentially beneficial effect of PRP. Nevertheless, the absence of a control group does not permit any final conclusion [27].

\section{Patellar tendinopathy (jumper's knee)}

Only three articles of moderate quality (level of evidence III and IV) investigated knee extensor lesions in a recent review on the subject [28].

The first study was performed on eight elite athletes in 2007. Participants received a single injection of PRP and were observed for four months, but only seven patients received a follow up examination. Nevertheless, the authors found a significant improvement of the VISA score and of the radiological aspects in magnetic resonance (tendon abnormal signal and pre-insertion edema) [29].

The second article, a pilot study, included twenty athletes who experienced recurrent patellar tendinopathy symptoms, over a twentymonth period. Participants received three PRP injections within one month. Pain was evaluated on a visual analog scale, sports activity on the Tegner scale. Results showed a significant improvement at six months, compared to baseline. At the end of the study, most athletes returned to the same competition level as before. No relevant side effects were noted, except for pain at the time of injection. The rehabilitation protocol after the infiltration was systematically structured, an element underscored by the authors as a key factor for the success described. These investigators claim that such standardization should be a prerequisite for all future studies. This study confirms the importance of the combination of growth factors and mechanical stimuli. Perhaps mechanical stimulation acts synergically with biological treatment and also modifies the micro-environment influencing tissue repair, independently of the presence of growth factors $[2,30]$.

The most recent study followed 15 patients with a jumper's knee resistant to conservative treatment. Participants' symptoms lasted for a mean of 24 months. All received multiple PRP injections. This population was then compared to 16 controls with moderate tendinopathy symptoms (mean duration of symptoms: six months), who had been admitted to a classical physiotherapy treatment program. Follow-up took place after six months. No difference in pain, patient satisfaction with management and time to return to sport was observed between both groups. However, the 2 groups were not randomized and the characteristics (severity and duration of symptoms) of the control group were not comparable with those of the intervention group. Those limitations should be taken into account when interpreting the study's results. Nevertheless, patients with refractory jumper's knee, resistant to conventional treatment, may respond to PRP injections as positively as patients with less severe pathologies and classically treated [31].

\section{Elbow tendinopathy}

Since 2003, only a few single case studies and case series reports were published on epicondylopathy, with encouraging results [5].

A prospective, pilot, cohort study investigated the effect of PRP infiltration in 15 patients with chronic elbow tendinopathies. PRP was administered under local anesthesia. The test group was compared to a control group of five patients receiving local anesthesia only. The patients were observed for 24 months. Unfortunately, by week eight, all of the small control group participants left the study because of the persistence of pain. At that time, $60 \%$ of the subjects who had been injected with PRP, but only $16 \%$ of those not treated with PRP, showed a significant decrease of pain. At six months, 80 of patients in the PRP group still showed a decrease in pain, and 93 at 24 months. Functional improvement was similar according to the Mayo elbow score. In addition, 94 of the participants returned to work or restarted their sports activities after 24 months [32]. 
These findings were confirmed in a recent, randomized, double blind controlled trial that included 51 patients in a PRP group, and 49 patients in a group that received local infiltrations of corticosteroids. Pain (VAS) and a functional score of the upper extremity (DASH score) improved significantly compared to baseline values in both groups without any significant difference between groups during the first weeks after injections. From week 12, pain decreased and DASH significantly improved in the PRP group only, and for up to one year. This confirms the potentially early effect of corticosteroids in insertion injuries during the first weeks after injection, but maybe also their subsequent, negative influence on the tendon healing process [33].

Recently, a double blinded, randomized trial investigated two types of blood injections. A group of 150 patients with elbow symptoms resistant to conventional treatment received either PRP injections or autologous conditioned plasma. The injections were repeated twice and both groups were compared. Unfortunately, there was no placebo control group. The authors of this study planned a follow up after six months. In both groups, they reported marked reduction in pain and increase in function, but without any significant difference between groups. This raises the question of which concentration of platelets and growth factors is the best. "Less might be better", and the fact that the tissue healing response could be saturated after a certain concentration of growth factors locally should be taken into account. The number of platelets and the concentration of growth factors applied to the lesion site are probably determinant factors. Also, after a certain stage of healing, other factors may start influencing the process [34].

\section{PRP in Acute Human Ligament Injuries}

Studies on ligaments have mainly been conducted on animals, as seen before, but there are also some case reports in humans some years ago. The complex process of normal tissue healing is only partially known. In addition, the temporal expression of cells and molecules during spontaneous healing has been barely studied and thus, the timing for optimal PRP administration is not yet known. No human studies are available about timing. A recent systematic review identifying articles with the keywords "PRP" and "ligament" included only three human studies on ACL reconstruction which fulfilled criteria for an evidence level I or II [23]. No statistically significant differences with regard to clinical outcomes, tunnel widening, and graft integration have been identified between control and PRP groups.

In general, studies on ACL reconstruction focus more on the osteo-integration of ACL grafts and less on the graft itself. However, a prospective, single blinded MRI study has recently shown a $48 \%$ reduction in the time needed to present a complete, homogeneous graft signal with PRP injection versus controls, after surgical ACL reconstruction [35].

As this article should particularly help primary care physicians and sports medicine specialists in the clinical decision making we kept attention on conservative treatment of ligament injuries. Forster et al. [6] evaluated professional soccer players $(n=22)$ with grade II acute medial collateral ligament injuries. A group of 22 was treated with a single PRP injection within 72 hours of injury and compared to a control group treated with standard rest and rehabilitation. The returnto-play time was shortened by $27 \%$ in the PRP treated group compared to the control group.

More recently, a published case report evaluated the effect of two consecutive injections of a preparation rich in growth factors (PRGF) on an acute rupture of the medial collateral ligament of the elbow of a judoka. The authors reported an early return to full contact training at 3 months after injury and faster MRI recovery images at 6 weeks follow-up, compared to "classical" MRI outcome of those injuries [36].

Thus, human studies other than those concerning ACL surgery are inconclusive regarding any clearly positive effect of PRP on ligament injuries. Further investigations are needed to assess the effectiveness of PRP injections in the treatment of such lesions.

\section{PRP in Acute Human Muscle Injuries}

Physiologically, muscle injuries undergo distinct but overlapping healing phases, and the proper timing of PRP application is still unclear. The speed of recovery depends on the severity of the lesion, the treatment applied after injury, and the patient's ability to heal soft tissue lesions. At the time of trauma, myofiber ruptures and capillary lesions result in hematoma that fills the gap, with the release of cytokines and growth factors. The inflammatory phase thus starts from the first day on, involves a number of inflammatory responses and after one week, leads to the regenerative process. During this time and up to four to six weeks, the muscle gap is progressively replaced by fibrotic tissue which provides early support for new muscle tissue, involving in particular fibroblasts. Capillary neo-formation occurs to reestablish the nutrition of the area. The proliferation and differentiation of muscle precursor cells then permit the fusion of differentiated cells, leading to the formation of new, multinucleated myotubes. After week three, the remodeling phase begins, leading to the increase of the muscle's functional capacities.

Regeneration frequently does not fully restore muscle tissue to its previous level. The two processes of healing and fibrosis compete with each other, impairing full regeneration. Thus, the most important issue in muscle regeneration could be to prevent the development of fibrosis, thereby avoiding the apparition of a fibrotic tissue scar. PRP, with its high TGF-Betal level, might be deleterious to muscle healing by inducing fibrotic, muscle scar formation, possibly leading to increased risk of re-injury [37,38].

Moreover, the presence of neutrophils in some PRP preparations raises the problem of the potential release of pro-inflammatory proteases and reactive oxygen species by the said cells, leading to secondary damage to muscle tissue [18].

Despite the relatively promising results in animal models, literature on humans is much less clear. No randomized controlled studies conducted in humans could be identified and only three other studies were performed on small samples with only few valuable controls. Therefore, statistical power to support clinical evidence in humans is generally weak, due to this lack of methodologically robust studies.

The first description in humans did not strictly use PRP, but rather autologous conditioned serum (ACS). Eighteen professional athletes suffering from muscle strains were treated by ACS muscle injection and compared with 11 athletes with similar injuries and previously treated by Actovegin or Traumeel injections. Primary outcome was the time to return to competitive sport. The study reported a significant reduction in return-to-field days (16 versus 22), but presented numerous limitations: small sample size, no-blinded, atypical controls, variable injury sites, absence of long-term follow-up [39].

Two other case-reports were published. The first one by Loo et al. [40] presented a 35-year-old male bodybuilder with an adductor longus strain injury confirmed by echography. A weekly intra-muscle injection for three consecutive weeks permitted the return to competitive training, only one week after the last injection. Nevertheless, there were no details about the grade of injury, additional treatments, and followup. 
Citation: Ziltener JL, Allet L, Sclison P, Grosclaude M (2012) How Effective are Injections of Platelet-Rich Plasma (PRP) for the Treatment of Sports Injuries: a Critical Review of the Literature. J Sport Medic Doping Studie S2:003. doi:10.4172/2161-0673.S2-003

Page 5 of 6

The second paper described a 42-year-old active male with a sudden history of grade II medial hamstring muscle strain (semi-membranous), confirmed by an MRI scan. PRP was injected once in the muscle, with an ultra-sound guided technique. Seventeen days after injury, the athlete had full ROM, was pain-free during muscle contractions and was able to perform all his recreational sporting activities. MRI performed at the same time revealed complete resolution of the signal enhancement, which was clearly different to the usual MRI outcome, with a signal intensity remaining positive for at least six weeks after injury. Longterm follow-up at twelve months did not reveal any negative outcomes [41].

Some unpublished case-series were also presented in different Sports Medicine congresses, but all the presentations were retrospective, without control groups, and comparisons were based on expected return-to-play delays according to previously published series [42].

\section{Concluding Remarks}

The role of PRP in tissue healing could open a new field in sports medicine, but a significant amount of research is still needed to understand the effect of PRP on the regenerative and healing processes of a given soft tissue.

Many basic science or animal studies assessed the effect of PRP on the musculoskeletal system during the last decade. In general results are promising. However, this is not yet the case for human studies, especially in the area of sports medicine. Only a few human studies could be identified and a paucity of RCTs providing level I or II evidence for the efficacy of this intervention method exists. Furthermore, several questions remain unanswered. First of all, there is no consensus about the PRP preparations. A large diversity exists without knowing which one to adopt as the clinical best practice. There are no recommendations concerning the number of injections that should be administered, a single one or serial injections. We further do not know if pre-injection anesthetic affects the outcome. No standardized procedure for the administration of the injections exist (ultrasound guidance or not for example) and no precise information about ideal timing to administer PRP, depending on the lesions. No standardized follow-up can be found, especially concerning the use of non-steroidal anti-inflammatory drugs. A comprehensive rehabilitation planning is actually unavailable, in terms of what kind of activities should be permitted or avoided and during how much time.

However, studies agree on the absence of side effects or complications, albeit with limited duration of follow-up. Thus, the usefulness of autologous blood injections (growth factor preparations) for the treatment of acute or chronic injuries of the human musculoskeletal system, in particular among athletes, but also for people who play sport as a hobby, is still controversial. An analysis of the existing literature in human research reveals a weak evidence for the effect of PRP applications in soft tissue injuries. At this time, robust studies, with a good methodological quality and representative sample size are urgently needed and should be conducted. The authors are however confident that new studies will emerge in the near future.

Primary care physicians and sports medicine specialists should, with respect to current medical ethics, be cautious with the use of PRP to treat athletic or sporting injuries. Nevertheless, this field of treatment seems promising in the arsenal of non-operative management of sport injuries and particularly the cost-benefit ratio could be in favor of such procedures.

\section{References}

1. Creaney L, Hamilton B (2008) Growth factor delivery methods in the management of sports injuries: the state of play. Br J Sports Med 42: 314-320.

2. Kon E, Filardo G, Di Martino A, Marcacci M (2011) Platelet-rich plasma (PRP) to treat sports injuries: evidence to support its use. Knee Surg Sports Traumato Arthrosc 19: 516-527.

3. Engebretsen L, Steffen K, Alsousou J, Anitua E, Bachl N, et al. (2010) IOC consensus paper on the use of platelet-rich plasma in sports medicine. $\mathrm{Br} J$ Sports Med 44: 1072-1081.

4. Borrione P, Di Gianfrancesco A, Pereira MT, Pigozzi F (2010) PRP in muscle healing. Am J Phys Med Rehabil 89: 854-861.

5. Kampa RJ, Connell DA (2010) Treatment of tendinopathy: is there a role for autologous whole blood and PRP injection? Int J Clin Pract 64: 1813-1823.

6. Foster TE, Puskas BL, Mandelbaum BR, Gerhardt MB, Rodeo SA (2009) Platelet rich plasma: from basic science to clinical applications. Am J Sports Med 37: 2259-2272

7. Kajikawa $Y$, Morihara T, Sakamoto H, Matsuda K, Oshima $Y$, et al. (2008) Platelet-Rich plasma enhances the initial mobilization of circulation-derived cells for tendon healing. J Cell Physiol 215: 837-845.

8. Schnabel LV, Mohammed HO, Miller BJ, McDermott WG, Jacobson MS, et al. (2007) Platelet rich plasma (PRP) enhances anabolic gene expression patterns in flexor digitorum superficialis tendons. J Orthop Res 25: 230-240.

9. Klein MB, Yalamanchi N, Pham H, Longaker MT, Chang J (2002) Flexor tendon healing in vitro: Effects of TGF-beta on tendon cell collagen production. J Hand Surg Am 27: 615-620.

10. Fallouh L, Nakagawa K, Sasho T, Arai M, Kitahara S, et al. (2010) Effects of autologous platelet-rich plasma on cell viability and collagen synthesis in injured human anterior cruciate ligament. J Bone Joint Surg Am 92: 2909-2916.

11. Saiga K, Furumatsu T, Yoshida A, Masuda S, Takihira S, et al. (2010) Combined use of bFGF and GDF-5 enhances the healing of medial collateral ligament injury. Biochem Biophys Res Commun 402: 329-334.

12. Hildebrand KA, Woo SL, Smith DW, Allen CR, Deie M, et al. (1998) The Effects of Platelet-Derived Growth Factor-BB on Healing of the Rabbit Medial Collatera Ligament. An in Vivo Study. Am J Sports Med 26: 549-554.

13. Letson AK, Dahners LE (1994) The Effect of Combinations of Growth Factors on Ligament Healing. Clin Orthop Relat Res 308: 207-212.

14. Batten ML, Hansen JC, Dahners LE (1996) Influence of Dosage and Timing of Application of Platelet-Derived Growth Factor on Early Healing of the Rat Medial Collateral Ligament. J Orthop Res 14: 736-741.

15. Kasemkijwattana C, Menetrey J, Bosch P, Somogyi G, Moreland MS, et al (2000) Use of growth factors to improve muscle healing after strain injury. Clin Orthop Relat Res 370: 272-285.

16. Lefaucheur JP, Gjata B, Lafont $\mathrm{H}$, Sebille A (1996) Angiogenic and inflammatory responses following skeletal muscle injury are altered by immune neutralization of endogenous basic fibroblast growth factor, insulin-like growth factor and transforming growth factor-beta 1. J Neuroimmunol 70: 37-44.

17. Wright-Carpenter T, Opolon P, Appell HJ, Meijer H, Wehling P, et al. (2004) Treatment of muscle injuries by local administration of autologous conditioned serum: Animal experiments using a muscle contusion model. Int J Sports Med 25: $582-587$

18. Hammond JW, Hinton RY, Curl LA, Muriel JM, Lovering RM (2009) Use of autologous PRP to treat muscle injuries. Am J Sports Med 37: 1135-1142.

19. Sharma P, Maffulli N (2005) Tendon injury and tendinopathy: healing and repair. J Bone Joint Surg Am 87: 187-202.

20. Maffulli N, Longo UG, Denaro V (2010) Novel approaches for the management of tendinopathy. J Bone Joint Surg Am 92: 2604-2613.

21. Andia I, Sanchez M, Maffulli N (2010) Tendon healing and PRP therapies. Expert Opin Biol Ther 10: 1415-1426.

22. De Vos RJ, Van Veldhoven PL, Moen MH, Weir A, Tol JL, et al. (2010) Autologous growth factor injections in chronic tendinopathy: a systematic review. Br Med Bull 95: 63-77.

23. Taylor DW, Petrera M, Hendry M, Theodoropoulos JS (2011) A Systematic Review of the Use of Platelet-Rich Plasma in Sports Medicine as a New Treatment for Tendon and Ligament Injuries. Clin J Sport Med 21: 344-352. 
Citation: Ziltener JL, Allet L, Sclison P, Grosclaude M (2012) How Effective are Injections of Platelet-Rich Plasma (PRP) for the Treatment of Sports Injuries: a Critical Review of the Literature. J Sport Medic Doping Studie S2:003. doi:10.4172/2161-0673.S2-003

24. De Vos RJ, Weir A, Verhaar J, Weinans H, Tol JL, et al. (2010) Platelet-rich plasma injection for chronic Achilles tendinopathy. JAMA 303: 144-149.

25. De Jonge S, De Vos RJ, Weir A, Verhaar J, Weinans H, et al. (2011) One year follow-up of PRP treatment in chronic Achilles tendinopathy. Am J Sports Med 39: 1623-1629.

26. De Vos RJ, Weir A, Tol JL, Verhaar J, Weinans H, et al. (2011) No effects of $\mathrm{PRP}$ on ultrasonographic tendon structure and neovascularisation in chronic midportion Achilles tendinopathy. Br J Sports Med 45: 387-392.

27. Gaweda K, Tarczynska M, Kryzanowski W (2010) Treatment of Achilles tendinopathy with PRP. Int J Sports Med 31: 577-583.

28. Van Ark M, Zwerver J, Akker-Scheek I (2011) Injection treatments for patellar tendinopathy. Br J Sports Med 45: 1068-1076.

29. Volpi P, Marinoni L, Bait C, De Girolamo L, Schoenhuber H (2007) Treatment of chronic patellar tendinosis with buffered PRP: a preliminary study. Med Sport 60: 595-603.

30. Kon E, Filardo G, Delcogliano M, Russo a, Bondi A, et al. (2009) Platelet-rich plasma: new clinical application. A pilot study for treatment of jumper's knee. Injury 40: 598-603.

31. Filardo G, Kon E, Della Villa S, Vincentelli F, Fornasari PM, et al. (2010) Use of platelet-rich plasma for the treatment of refractory jumper's knee. Int Orthop 34: 909-915

32. Mishra A, Pavelko T (2006) Treatment of chronic elbow tendinosis with buffered platelet-rich plasma. Am J Sports Med 34: 1774-1778.

33. Peerbooms JC, Sluimer J, Bruijn DJ, Gosens T (2010) Positive effects of an autologous platelet concentrate in lateral epicondylitis in a double- blind randomized controlled trial: PRP versus corticosteroid injection with 1 year follow-up. Am J Sports Med 38: 255-262.
34. Creaney L, Wallace A, Curtis M, Connell D (2011) Growth factor-based therapies provide additional benefit beyond physical therapy in resistant elbow tendinopathy: a prospective randomized trial of autologous blood injections versus PRP injections. Br J Sports Med 45: 966-971.

35. Radice F, Yanez R, Gutierrez V, Rosales J, Pinedo M, et al. (2010) Comparison of Magnetic Resonance Imaging Findings in Anterior Cruciate Ligament Grafts with and Without Autologous Platelet-Derived Growth Factors. Arthroscopy 26 50-57.

36. Mei-Dan O, Carmont M, Kots E, Barchilon V, Nyska M, et al. (2010) Early Return to Play following Complete Rupture of the Medial Collateral Ligament of the Elbow Using Preparation Rich in Growth Factors: A Case Report. J Shoulder Elbow Surg 19: e1-5.

37. Chan YS, Li Y, Foster W, Fu FH, Huard J (2005) The use of suramin, an antifibrotic agent, to improve muscle recovery after strain injury. Am J Sports Med 33: 43-51.

38. Sampson S, Gerhardt M, Mandelbaum B (2008) Platelet rich plasma injection grafts for musculoskeletal injuries: A review. Curr Rev Musculoskelet Med 1 : 165-174.

39. Wright-Carpenter T, Klein P, Schafferhof P, Appell HJ, Mir LM, et al. (2004) Treatment of muscle injuries by local administration of autologous conditioned serum: a pilot study on sportsmen with muscle strains. Int J Sports Med 25 588-593.

40. Loo W, Lee D, Soon M (2009) Plasma rich in growth factors to treat Adductor Longus tear. Ann Acad Med 38: 733-734.

41. Hamilton BH, Knez W, Eirale C, Chalabi H (2010) Platelet enriched plasma for acute muscle injury. Acta Orthop Belg 76: 443-448.

42. Hamilton BH, Best TM (2011) Platelet enriched plasma and muscle strain injuries: challenges imposed by the burden of proof. Clin J Sport Med 21: 31-36.

This article was originally published in a special issue, Sport Management handled by Editor(s). Dr. Kamal Bali, Epworth Hospital, Australia 